Supporting Information for

\title{
Soft Plasmonic Assemblies Exhibiting Unnaturally High Refractive Index
}

Ji-Hyeok Huh', Jaewon Lee', and Seungwoo Lee ${ }^{1,2,3 *}$

IKU-KIST Graduate School of Converging Science and Technology, Korea University, Seoul 0284I, Republic of Korea

2Department of Biomicrosystem Technology, Korea University, Seoul 0284I, Republic of Korea

${ }^{3}$ KU Photonics Center, Korea University, Seoul 0284I, Republic of Korea

*Email: seungwoo@korea.ac.kr

Keywords: Self-assembly, Plasmonic colloids, Refractive index, Metamaterials, Effective medium theory

Contents:

1. Synthesis and quantitative analysis of gold $(\mathrm{Au})$ colloids

2. Analysis of optical microscope images and reflectance spectra.

3. s-parameter retrieval method and electromagnetic field distribution analysis

4. Ellipsometer measurement

5. Modal analyses of self-assembled optical metamaterials

6. Effective parameters

7. Synthetic route for Au colloids used in this study

8. Assembly route for Au NC monolayer 


\section{Synthesis and quantitative analysis of gold $(\mathrm{Au})$ colloids}

\section{Synthesis protocol}

First, Au nanorods (Au NRs) were synthesized as follows. A total of $125 \mu \mathrm{L}$ of $10 \mathrm{mM}$ gold (III) chloride trihydrate $\left(\mathrm{HAuCl}_{4}\right)$ was added to $5 \mathrm{~mL}$ of $100 \mathrm{mM}$ cetyltrimethylammonium bromide (CTAB) aqueous solution. $300 \mu \mathrm{L}$ of $10 \mathrm{mM}$ ice-cold sodium borohydride (NaBH4) was rapidly injected into the aqueous solution with vigorous stirring for few minutes to nucleate seeds. The seed solution was incubated in a $28^{\circ} \mathrm{C}$ water bath for $30 \mathrm{~min}$. Then, $200 \mathrm{~mL}$ of $100 \mathrm{mM}$ CTAB, $10 \mathrm{~mL}$ of $10 \mathrm{mM} \mathrm{HAuCl}_{4}, 1.8 \mathrm{~mL}$ of $10 \mathrm{mM}$ silver nitrate (AgNO3), $1.14 \mathrm{~mL}$ of $100 \mathrm{mM} \mathrm{L}-$ ascorbic acid (AA), and $240 \mu \mathrm{L}$ of seed solution were added and stirred for $1 \mathrm{~min}$ to synthesize $\mathrm{Au}$ NRs. The as-synthesized Au NR solution was then centrifuged two times for 15 minutes at 9,000 rpm and resuspension in $50 \mathrm{mM} \mathrm{CTAB}$ aqueous solution. To selectively etch the tips (high energy sight) of the Au NRs, theses solution was brought to 2 optical density (O.D.) with $50 \mathrm{mM}$ CTAB aqueous solution. They were mixed with $90 \mu \mathrm{M} \mathrm{HAuCl}_{4}$ aqueous solution and gently stirred for 4 hours at $40{ }^{\circ} \mathrm{C}$. Au NRs were transformed into $20 \mathrm{~nm}$ Au nanospheres (NSs). The Au NSs were centrifuged two times for $30 \mathrm{~min}$ at $11,000 \mathrm{rpm}$ in $100 \mathrm{mM}$ cetylpyridinium chloride (CPC) aqueous solution.

These $20 \mathrm{~nm} \mathrm{Au} \mathrm{NSs} \mathrm{were} \mathrm{used} \mathrm{as} \mathrm{seeds.} \mathrm{First,} 20 \mathrm{~mL}$ of $10 \mathrm{mM} \mathrm{CPC}, 350 \mu \mathrm{L}$ of $10 \mathrm{mM}$ $\mathrm{HAuCl}_{4}, 4.5 \mathrm{~mL}$ of $100 \mathrm{mM} \mathrm{AA}$ and $6 \mathrm{~mL}$ of seeds (at 1 O.D.) were mixed in deionized (DI) water. Au NS seeds grew into Au concaved rhombic dodecahedras (Au CRDs) and then these assynthesized $\mathrm{Au} \mathrm{CRDs}$ were washed with $50 \mathrm{mM} \mathrm{CTAB}$ aqueous solution. $\mathrm{Next}, \mathrm{HAuCl}_{4}$ aqueous solution was added to the Au CRDs solution and gently stirred for 4 hours at $40{ }^{\circ} \mathrm{C}$ to selectively etch the $\mathrm{Au}$ CRDs vertices (i.e., oxidative dissolution). These reductive growths and etching processes were repeated until the etched Au CRDs (Au nanospheres; Au NSs) aspect ratio was reduced to $\sim 1.0$.

By using these $\mathrm{Au}$ NSs (etched Au CRDs) as seeds, uniform and high yield Au NCs were synthesized. Briefly, $75 \mathrm{~mL}$ of $100 \mathrm{mM} \mathrm{CPC}, 500 \mu \mathrm{L}$ of $100 \mathrm{mM}$ potassium bromides $(\mathrm{KBr}), 100$ $\mu \mathrm{L}$ of $10 \mathrm{mM} \mathrm{HAuCl}_{4}$, and $150 \mu \mathrm{L}$ of $100 \mathrm{mM}$ AA were mixed with $1.5 \mathrm{~mL}$ and $6 \mathrm{~mL}$ seeds $(1$ O.D.) each for $85 \mathrm{~nm}$ and $60 \mathrm{~nm}$ Au NCs.

\section{Quantitative analysis}

We used the custom-built algorithmic script for quantitatively evaluating the SEM images of synthesized Au NCs. The algorithm can functionalize the centroid to edge distance as $d(\theta)$ and it is used for quantitation of aspect ratio of Au NCs. In addition, the radii of curvatures at each edge of $\mathrm{Au}$ NCs were determined at the inflection point of $d(\theta)$. 


\section{Analysis of optical microscopy images and reflectance spectra.}

The optical microscopy images and reflectance spectra were collected by using a custom-built optical microscope spectrometer, in which both the imaging spectrometer (IsoPlane, Princeton Instruments) and CCD (PIXIS-400B, Princeton Instruments) were integrated into an optical microscope (Eclipse Ni-U, Nikon). A broadband light was illuminated by $12 \mathrm{~V} / 100 \mathrm{~mW}$ halogen lamp. The source light was polarized to be $s$-pol. Reflected light was collected with a $10 \times$ objective lens with a numerical aperture of 0.3 .

3. s-parameter retrieval method and electromagnetic field distribution analysis

s-parameter retrieval method

The numerical simulation was carried out by both finite-element method (FEM). All effective parameters in this study were calculated by using the $s$-parameter retrieval technique described in Smith et al. ${ }^{1}$ To set up the simulation modelling for Au NC monolayer, lateral dimension of cubical unit cell was determined as size of Au NC plus the gap distance. The $x z$ and $y z$ plane were taken as periodic boundary condition and free space region was defined as $20 \mu \mathrm{m}$. The plane wave propagates along the $\mathrm{z}$-axis direction and was polarized to $\mathrm{x}$-axis. The dielectric constants of $\mathrm{Au}$ were taken from Johnson and Christy. ${ }^{2}$ The calculated scattering coefficients (amplitude and phase of transmission/reflection coefficient; $S_{11}, S_{21}$ ) were used for extracting the effective parameters (by using $s$-parameter retrieval technique).

\section{Electromagnetic field distribution analysis}

To calculate electromagnetic fields distribution inside a unit cell, the FEM simulator was utilized. The electric field distributions were collected at the center of the gap between Au NCs and surface charge densities were plotted at the surface of the Au NCs. The magnetic field spatial distributions were collected at the center of the Au NCs and normalized by incident electromagnetic field.

\section{Ellipsometer measurement}

An ellipsometer (J.A. Woollam, VASE \& IR-VASE Mark II Ellipsometer) was utilized to obtain the effective refractive indices of the Au NCs monolayer from the near-infrared to mid-infrared regimes. At the $70^{\circ}$ tilting angle measuring mode was selected and the beam spot size of the light source was $8 \mathrm{~mm}^{2}$. Before the effective parameter (refractive index) measurements, the refractive indices of the glass substrate without $\mathrm{Au}$ NCs monolayer was measured. The effective refractive indices and the thickness of the Au NCs monolayer on the glass substrate were measured by the b-spline model. 


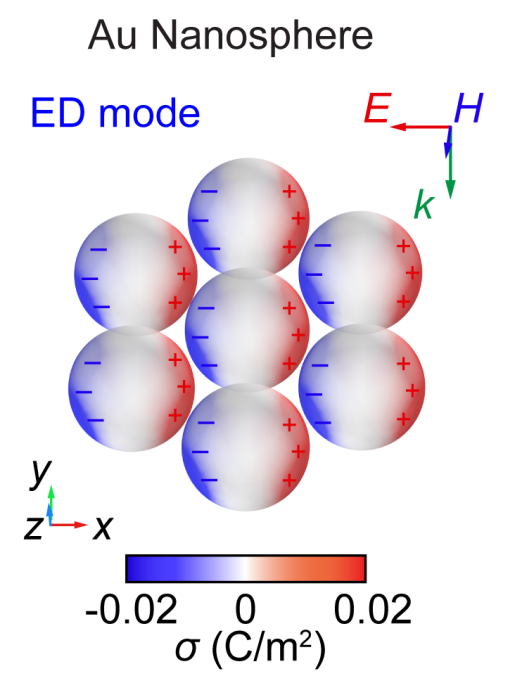

Au Nanooctahedral
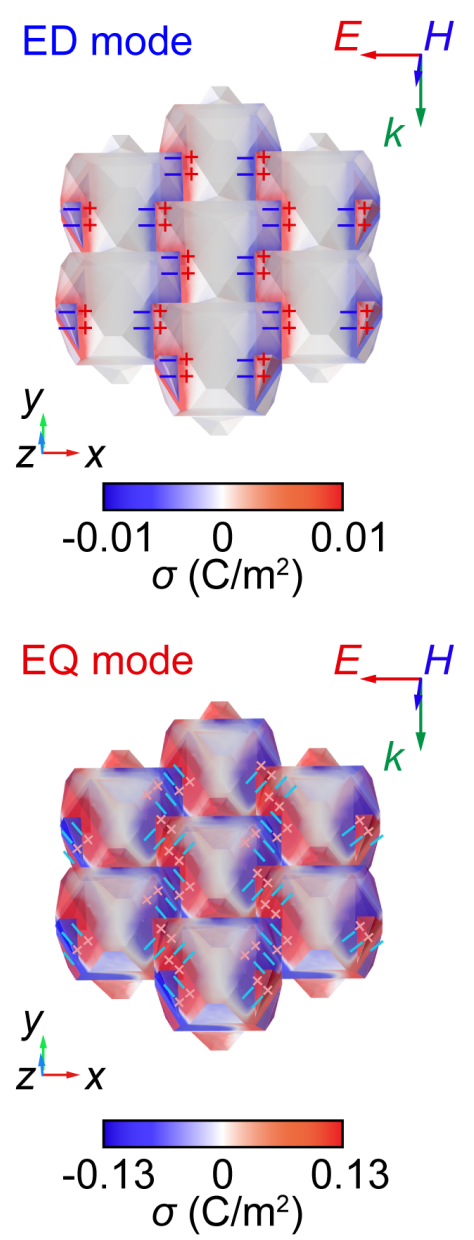

Au Nanocube
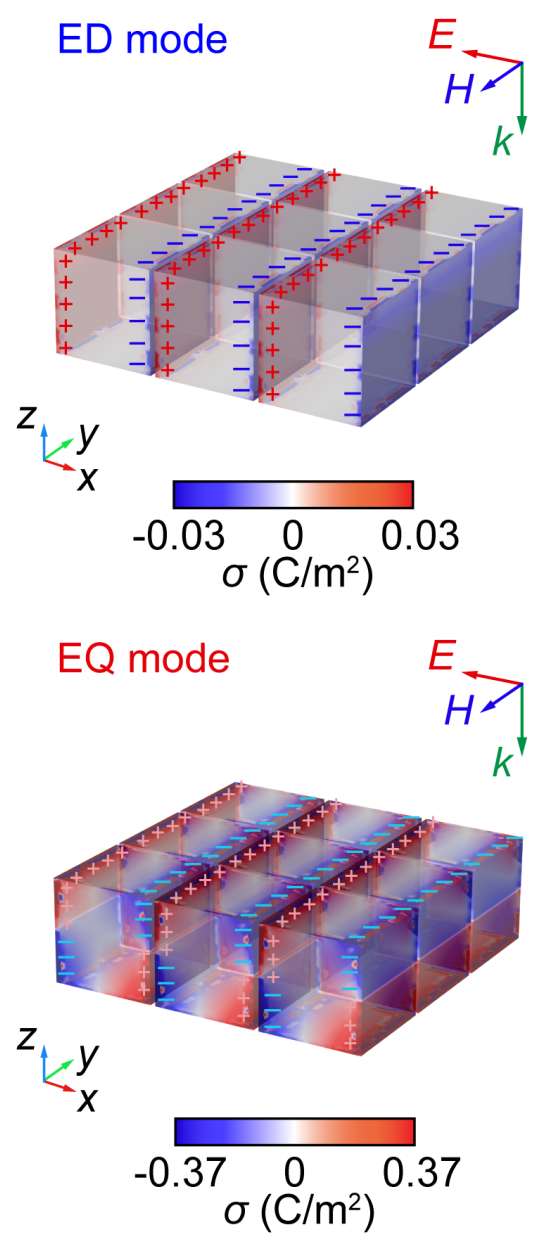

Figure. S1 Modal analyses of electric dipolar (ED) and quadrupolar (EQ) resonances of Au NS, NO, and NC optical metamaterials. Charge distributions of the 60-nm Au NS, NO, and NC monolayer (gap: $3 \mathrm{~nm}$ ) at the ED and EQ resonant wavelength (predicted by using the FEM). EQ mode is not available for Au NS monolayer at the wavelength of interest. 


\section{Effective parameters}
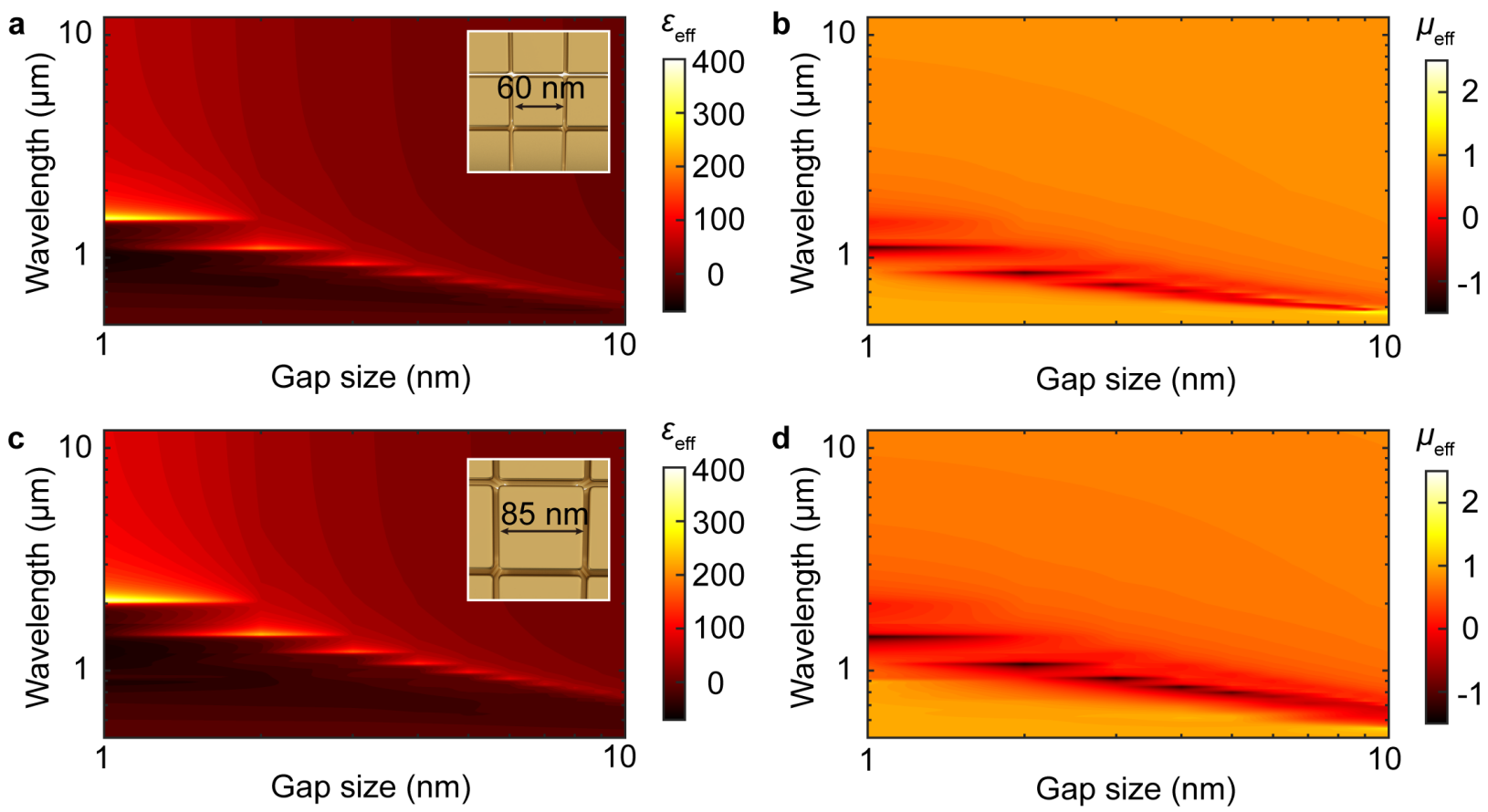

Figure. S2 (a) Effective permittivity $\left(\varepsilon_{\text {eff }}\right)$ and (b) permeability $\left(\mu_{\text {eff }}\right)$ of the 60 -nm Au NC monolayers with various gaps (1-10 nm). (c) $\varepsilon_{\text {eff }}$ and (d) $\mu_{\text {eff }}$ of the $85-\mathrm{nm}$ Au NC monolayers with various gaps $(1-10 \mathrm{~nm})$. 
a

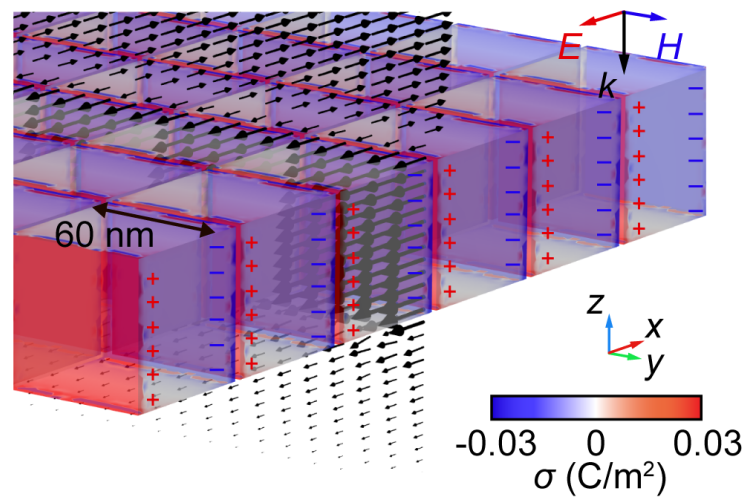

b

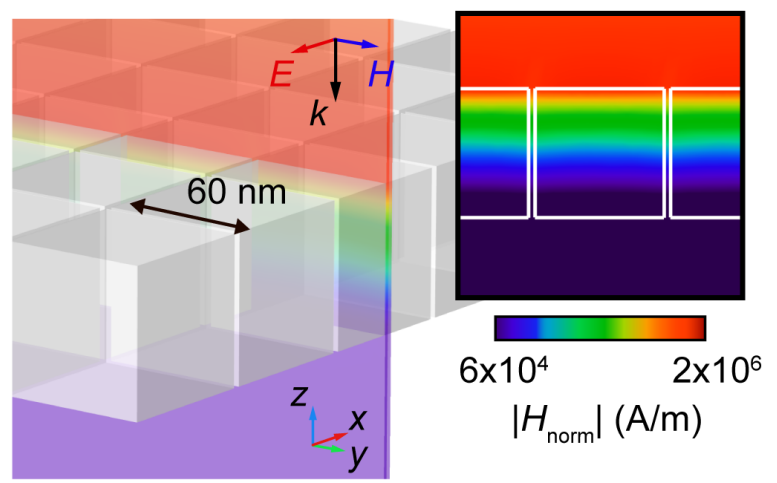

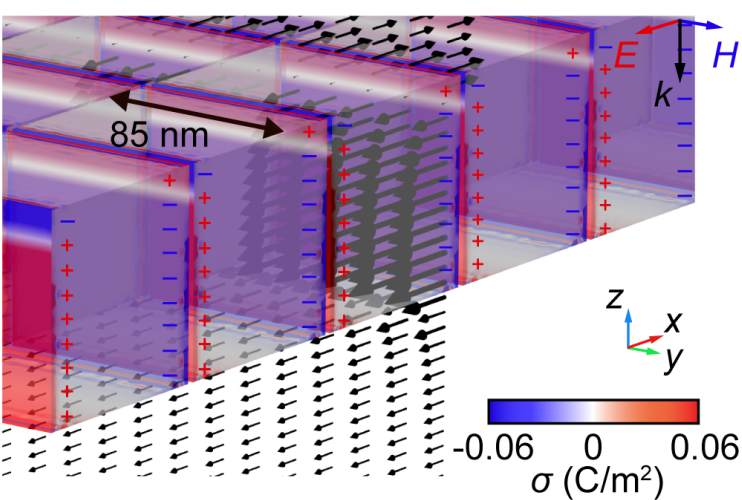
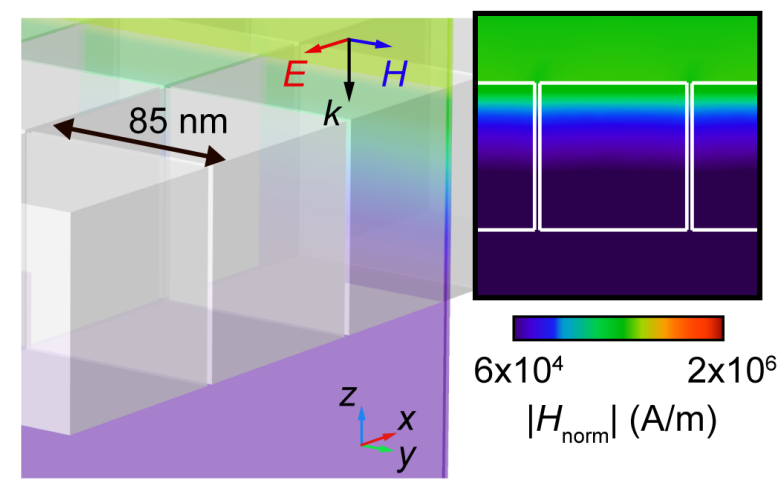

Figure. S3 (a) Electric field (black arrows) and charge distributions of the 60- and 85-nm Au NC monolayers (gap: $4 \mathrm{~nm}$ ) at the ED resonant wavelength (predicted by using the FEM). (b) Magnetic field intensity distributions of the 60- and 85-nm Au NC monolayers (gap: $4 \mathrm{~nm}$ ) at the ED resonant wavelength (predicted by using the FEM). 


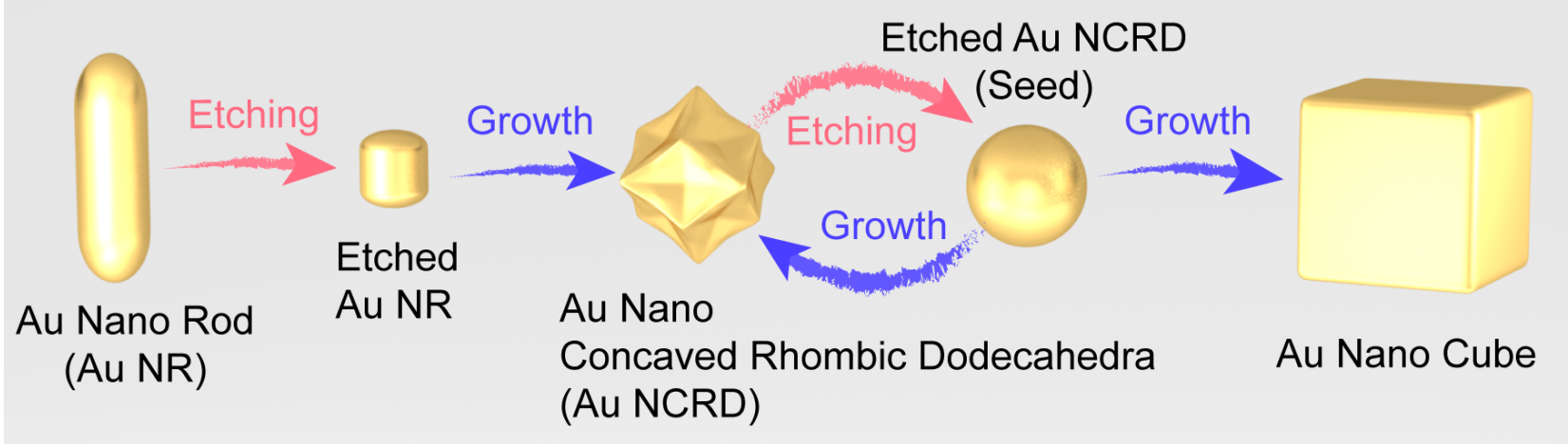

Figure. S4 Schematic of the synthesis of highly uniform Au NCs.

8. Assembly route for Au NC monolayer

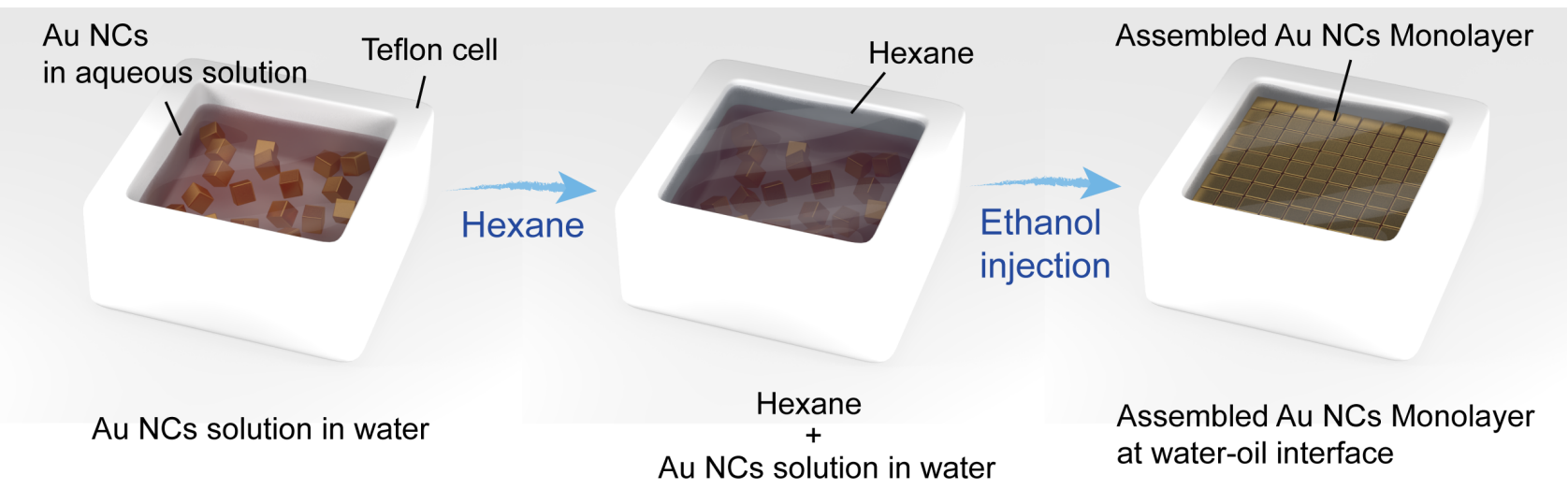

Figure. S5 Schematic of the interfacial self-assembly of Au NCs.

\section{References}

1. Smith, D. R.; Vier, D. C.; Koschny, T.; Soukoulis, C. M. Electromagnetic Parameter Retrieval from Inhomogeneous Metamaterials. Phys. Rev. E 2005, 71, 036617.

2. Johnson, P. B.; Christy, R. W. Optical Constants of Noble Metals. Phys. Rev. B 1972, 6, 4370. 